Editor's Note: These short, critical reviews of recent papers in the Journal, written exclusively by graduate students or postdoctoral fellows, are intended to summarize the important findings of the paper and provide additional insight and commentary. For more information on the format and purpose of the Journal Club, please see http://www.jneurosci.org/misc/ifa_features.shtml.

\title{
Heat Shock Protein 90 Modulates LRRK2 Stability: Potential Implications for Parkinson's Disease Treatment
}

\author{
Andrés Hurtado-Lorenzo* and Vasanti S. Anand ${ }^{\star}$ \\ Neuroscience Discovery Research, Wyeth Research, Princeton, New Jersey 08543 \\ Review of Wang et al. (http://www.jneurosci.org/cgi/content/full/28/13/3384)
}

Parkinson's disease (PD) is a neurological disorder in which degeneration of midbrain dopaminergic neurons leads to severe motor impairment. Neurotransmitter replacement therapy using the dopamine precursor levodopa remains the gold standard symptomatic treatment of the disease. This treatment, although effective, results in motor complications, such as dyskinesias. As an alternative, long-lasting dopamine agonists and nondopaminergic agents have been developed that can improve motor function and reduce the incidence of motor complications (Schapira et al., 2006). However, despite the advance in generating symptomatic treatments, the development of disease-modifying drugs that prevent, ameliorate, or reverse neuronal degeneration is the biggest unmet medical need.

The discovery of several mutations in different loci linked to familial PD has opened new avenues in the understanding of the disease, with a concomitant increase in opportunities for therapeutic intervention and disease modification. In particular, mutations in the recently discovered leucine-rich repeat 2 (LRRK2)

\footnotetext{
Received April 28, 2008; revised June 2, 2008; accepted June 3, 2008.

This work was supported by the Michael J. Fox Foundation. We thank Drs. Peter Reinhart, Steve Braithwaite, Robert Martone, and Warren Hirst from Wyeth Neuroscience for critical reading of this manuscript.

*A.H.-L. and V.S.A. contributed equally to this work.

Correspondence should be addressed to Dr. Andrés Hurtado-Lorenzo, Neuroscience Discovery Research, Wyeth Research, CN 8000, Princeton, NJ 08543. E-mail:hurtada2@wyeth.com.

DOI:10.1523/JNEUROSCI.1870-08.2008

Copyright $\odot 2008$ Society for Neuroscience $\quad$ 0270-6474/08/286757-003\$15.00/0
}

gene are now known to be the most common cause of autosomal-dominant PD, and the occurrence of LRRK2 mutations in patients without an overt family history, who were initially diagnosed with idiopathic PD, has also been documented (Klein and Schlossmacher, 2007).

$L R R K 2$ encodes a $286 \mathrm{kDa}$ protein of unknown function that contains five predicted functional domains, including a GTPase and kinase enzymatic domains. The most common mutation linked to PD is the glycine-to-serine substitution at position 2019 (G2019S), which is associated with a neurotoxic gain of function of the kinase activity (Taylor et al., 2006). Thus, efforts to identify specific kinase inhibitors have been initiated in a number of laboratories.

As an alternative to kinase inhibitors, Wang et al. (2008) demonstrated in a recent issue of The Journal of Neuroscience the feasibility of indirectly downregulating the augmented kinase activity of mutant G2019S LRRK2 by promoting its proteasomal degradation. This was achieved by inhibiting the chaperone activity of the heat shock protein 90 (Hsp90), a binding partner of LRRK2. Hsp90 is a member of a multichaperone complex involved in folding, activation, and stabilization of a set of proteins commonly referred to as Hsp90 client proteins. Although client proteins, Hsp90, and cdc37 (an Hsp90 cochaperone cell division cycle 37 protein) interact to form a stable complex, inhibition of the ATP-dependent function of Hsp90 promotes the association of client proteins with other chaperones, such as Hsp70, leading to proteasomal degradation (Stravopodis et al., 2007).

In their study, Wang et al. (2008) used brain extracts from conditional transgenic mice overexpressing G2019S LRRK2, and showed that the mutant LRRK2 formed a stable complex with Hsp90 and cdc37 in vivo [Wang et al. (2008), their Fig. 1B,C (http://www. jneurosci.org/cgi/content/full/28/13/ $3384 / F 1)]$. They also demonstrated that the chaperone function of Hsp90 was necessary for the formation of the LRRK2/ Hsp90 complex, because incubation of HEK-293 cells overexpressing mutant or wild-type LRRK2 with geldanamycin or PU-H71, two specific inhibitors of Hsp90, resulted in the dissociation of LRRK2 from Hsp90 and promoted its interaction with Hsc70, a constitutive isoform of the Hsp70 family [Wang et al. (2008), their Fig. $2 A$ (http://www.jneurosci.org/cgi/ content/full/28/13/3384/F2)]. More importantly, inhibiting Hsp90 in primary cortical neurons derived from G2019S transgenic mice [Wang et al. (2008), their Fig. 4A-D (http://www.jneurosci.org/cgi/ content/full/28/13/3384/F4)] or in HEK293 cells transiently transfected with wild-type and G2019S mutant LRRK2 [Wang et al. (2008), their Figs. 3A-C (http://www.jneurosci.org/cgi/content/ full/28/13/3384/F3), 5A-C (http://www. jneurosci.org/cgi/content/full/28/13/ $3384 /$ F5)] resulted in a significant decline of mutant and wild-type LRRK2 proteins, 
indicating that Hsp90 is crucial for maintaining LRRK2 stability.

Subsequently, the authors demonstrated that the decreased levels of LRRK2 mutant after Hsp90 inhibition are attributable to degradation of LRRK2 via the proteasomal pathway but not via the lysosomal pathway [Wang et al. (2008), their Fig. $6 A$ (http://www.jneurosci.org/cgi/ content/full/28/13/3384/F6)].

Last, as proof of principle that Hsp90 inhibition may rescue neurons from the neurotoxic effect of G2019S mutation, cortical neurons derived from transgenic mice were treated with the specific inhibitor PU-H71. This resulted in recovery of the axonal growth retardation that was associated with overexpression of G2019S LRRK2 [Wang et al. (2008), their Fig. $7 B, D, E \quad$ (http://www.jneurosci.org/cgi/ content/full/28/13/3384/F7)]

The work of Wang et al. (2008) provides both a new insight into the biology of LRRK2 and a proof of principle that the levels and the neurotoxic activity of G2019S LRRK2 can be downregulated by pharmacologically disrupting its interaction with Hsp90 (Fig. 1). At the same time, this work raises important questions about the potential therapeutic application of such a strategy, in particular, regarding the specificity of this approach, because LRRK2 is not the only Hsp90 client protein. Results from the cancer field indicate that specificity issues may be overcome because of a particular selectivity of Hsp90 inhibitors for tumor cells (Solit and Chiosis, 2008). However, because there is no evidence that Hsp90 inhibitors act exclusively in dopaminergic neurons undergoing neurodegeneration or in areas of the brain that express high levels of LRRK2 (e.g., striatum), evaluating off-target effects of these inhibitors, for instance, on global protein expression levels or other kinases in neurons, needs a closer examination. The authors suggest that inhibition of Hsp90 specifically downregulates LRRK2 and does not affect the overall steady-state levels of cellular proteins, because the levels of $\alpha$-synuclein, the main protein found in Lewy bodies (the pathological hallmark of $\mathrm{PD})$, remained unaltered [Wang et al. (2008), their Fig. 3D (http://www. jneurosci.org/cgi/content/full/28/13/ 3384/F3)]. Although mutant $\alpha$-synuclein was evaluated given its relevance in PD, the issue of whether Hsp90 inhibition alters overall protein levels remains to be established. Because $\alpha$-synuclein is prone to aggregation, clearance of this protein by the narrow proteasomal barrels can be

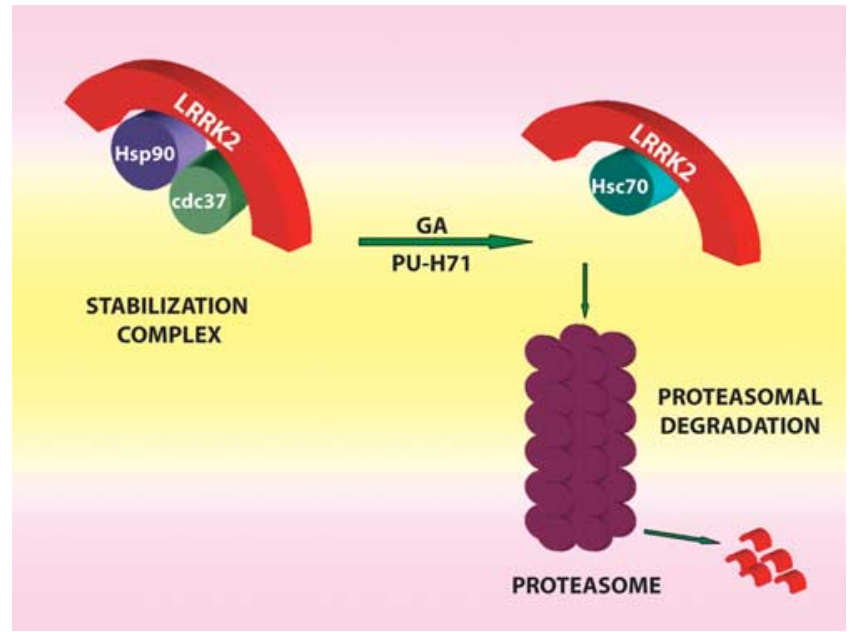

Figure 1. Model of Hsp90-mediated LRRK2 stability. Hsp90 - cdc37 complex binds to and stabilizes LRRK2 protein. Inhibition of $\mathrm{Hsp} 90$ chaperone activity with geldanamycin (GA) or PU-H71 disrupts these interactions and promotes the binding of LRRK2 to $\mathrm{Hsc} 70$, leading to proteasomal degradation of LRRK2.

inefficient, and because overexpression of mutant $\alpha$-synuclein leads to proteasomal dysfunction (Snyder et al., 2003), $\alpha$-synuclein may not be the ideal protein candidate to test the effects of Hsp90 inhibition on global steady-state protein levels. Hence, further analysis might be required using relevant proteins that do not interfere with or are resistant to proteasomal degradation.

Numerous kinases have been shown to be Hsp90 clients, and thus inhibition of this chaperone has emerged as a potential therapeutic approach when dysregulated kinase activity leads to disease (Sreedhar et al., 2004). Consequently, another issue that warrants further investigation is whether in the LRRK2 transgenic neuronal system used by Wang et al. (2008), Hsp90 inhibition leads to concomitant decreased levels of other protein kinases. Importantly, this could result in amplification of the potential therapeutic effects of Hsp90 inhibitors if proapoptotic kinases (e.g., JNK) are also depleted. However, if antiapoptotic kinases (e.g., Akt) are degraded as well, Hsp90 inhibition may render neurons more vulnerable to LRRK2-independent neurodegeneration.

A further question to be addressed is whether Hsp90 inhibition is effective in vertebrate models of PD. In this regard, work in the MPTP (1-methyl-4-phenyl1,2,3,6-tetrahydropyridine) mouse model of PD has demonstrated that intracerebral ventricular administration of the specific Hsp90 inhibitor geldanamycin prevents striatal dopamine depletion and denervation (Shen et al., 2005). However, the lack of a neurodegenerative or behavioral phenotype in the adult transgenic mice over- expressing G2019S LRRK2 used by Wang et al. (2008), and also the lack of a control transgenic mice overexpressing WT LRRK2, puts into question the relevance of Hsp90 inhibition in this system. In their study, defective axonal growth was only evident in cortical neurons derived from G2019S LRRK2 transgenic pups at postnatal day 1 . Thus, the generation of improved LRRK2 vertebrate models with adult neuropathological phenotype, characterized by neurodegeneration and/or motor impairment as reported in transgenic G2019S Drosophila (Liu et al., 2008), are urgently needed to evaluate this and other pharmacological manipulations of mutant LRRK2. In addition, it would be useful to determine whether in ventral mesencephalon cultures, dopaminergic neurons from transgenic G2019S mice also undergo axonal growth retardation.

Evidence is accumulating that inhibitors of Hsp90 are promising drug candidates for the treatment of diverse diseases. The clinical application of the specific inhibitor geldanamycin has been limited because of hepatic toxicity, but less toxic derivates of this drug have been identified and are currently in clinical trials for cancer (Solit and Chiosis, 2008). In addition to their toxicity, another limitation of Hsp90 inhibitors for the treatment of CNS disorders is their limited ability to cross the blood-brain barrier. Therefore, if Hsp90 inhibition is to become a realistic pharmacological approach for the treatment of PD, it is necessary to identify inhibitors capable of effectively penetrating the brain. In this regard, previous independent studies have demonstrated that systemic administration of Hsp90 inhibi- 
tors PU-DZ8 and EC102 reduced the accumulation of tau protein in a mouse model of tauopathies (Dickey et al., 2007; Luo et al., 2007). Whether the purine derivative PUH71 used by Wang et al. (2008) efficiently crosses the blood-brain barrier and is able to prevent the accumulation of LRRK2 and increased kinase activity and revert or prevent any associated neuropathology in vivo remains to be determined.

In conclusion, Wang et al. (2008) demonstrated that LRRK2, like other kinases, requires Hsp90 for its stabilization. Disrupting this interaction using Hsp90 inhibitors results in LRRK2 proteasomal degradation and concomitant depletion of the increased kinase activity of the G2019S mutation associated with PD. This approach, although still in its infancy, adds to the repertoire of pharmacological strategies that may eventually be used to regulate the neurotoxicity associated with mutant forms of LRRK2 and to modify the course of PD.

\section{References}

Dickey CA, Kamal A, Lundgren K, Klosak N, Bailey RM, Dunmore J, Ash P, Shoraka S, Zlat- kovic J, Eckman CB, Patterson C, Dickson DW, Nahman NS Jr, Hutton M, Burrows F, Petrucelli L (2007) The high-affinity HSP90-CHIP complex recognizes and selectively degrades phosphorylated tau client proteins. J Clin Invest 117:648-658.

Klein C, Schlossmacher MG (2007) Parkinson disease, 10 years after its genetic revolution: multiple clues to a complex disorder. Neurology 69:2093-2104.

Liu Z, Wang X, Yu Y, Li X, Wang T, Jiang H, Ren Q, Jiao Y, Sawa A, Moran T, Ross CA, Montell C, Smith WW (2008) A Drosophila model for LRRK2-linked parkinsonism. Proc Natl Acad Sci USA 105:2693-2698.

Luo W, Dou F, Rodina A, Chip S, Kim J, Zhao Q, Moulick K, Aguirre J, Wu N, Greengard P, Chiosis G (2007) Roles of heat-shock protein 90 in maintaining and facilitating the neurodegenerative phenotype in tauopathies. Proc Natl Acad Sci U S A 104:9511-9516.

Schapira AH, Bezard E, Brotchie J, Calon F, Collingridge GL, Ferger B, Hengerer B, Hirsch E, Jenner P, Le Novère N, Obeso JA, Schwarzschild MA, Spampinato U, Davidai G (2006) Novel pharmacological targets for the treatment of Parkinson's disease. Nat Rev Drug Discov 5:845-854.

Shen HY, He JC, Wang Y, Huang QY, Chen JF (2005) Geldanamycin induces heat shock protein 70 and protects against MPTP- induced dopaminergic neurotoxicity in mice J Biol Chem 280:39962-39969.

Snyder H, Mensah K, Theisler C, Lee J, Matouschek A, Wolozin B (2003) Aggregated and monomeric alpha-synuclein bind to the $\mathrm{S}^{\prime}$ proteasomal protein and inhibit proteasomal function. J Biol Chem 278:11753-11759.

Solit DB, Chiosis G (2008) Development and application of Hsp90 inhibitors. Drug Discov Today 13:38-43.

Sreedhar AS, Soti C, Csermely P (2004) Inhibition of Hsp90: a new strategy for inhibiting protein kinases. Biochim Biophys Acta 1697:233-242.

Stravopodis DJ, Margaritis LH, Voutsinas GE (2007) Drug-mediated targeted disruption of multiple protein activities through functional inhibition of the Hsp90 chaperone complex. Curr Med Chem 14:3122-3138.

Taylor JP, Mata IF, Farrer MJ (2006) LRRK2: a common pathway for parkinsonism, pathogenesis and prevention? Trends Mol Med 12:76-82.

Wang L, Xie C, Greggio E, Parisiadou L, Shim H, Sun L, Chandran J, Lin X, Lai C, Yang WJ Moore DJ, Dawson TM, Dawson VL, Chiosis G, Cookson MR, Cai H (2008) The chaperone activity of heat shock protein 90 is critical for maintaining the stability of leucine-rich repeat kinase 2. J Neurosci 28:3384-3391. 